\title{
Structural phase transition in two-dimensional tetramer-cuprate $\mathrm{Na}_{5} \mathrm{RbCu}_{4}\left(\mathrm{AsO}_{4}\right)_{4} \mathrm{Cl}_{2}$
}

\author{
V. Gnezdilov ${ }^{1}$, V. Bedarev¹, Yu. Pashkevich² ${ }^{2}$ P. Lemmens ${ }^{3}$, S. Zvyagin ${ }^{4}$, \\ S. Gnatchenko ${ }^{1}$, M. Pashchenko ${ }^{1}$, X. Mo ${ }^{5}$, W. Queen $^{5}$, and S.-J. Hwu ${ }^{5}$ \\ ${ }^{1}$ B. Verkin Institute for Low Temperature Physics and Engineering of the National Academy of Sciences of Ukraine \\ 47 Lenin Ave., Kharkov 61103, Ukraine \\ E-mail: gnezdilov@ilt.kharkov.ua \\ ${ }^{2}$ A. Galkin Donetsk Physico-Technical Institute of the National Academy of Sciences of Ukraine \\ 72 R. Luxemburg Str., Donetsk 83114, Ukraine \\ ${ }^{3}$ Institute for Physics of Condensed Matter, TU Braunschweig, Braunschweig D-38106, Germany \\ ${ }^{4}$ Hochfeld-Magnetlabor, Forschunszentrum Rossendorf, 01328 Dresden, Germany \\ ${ }^{5}$ Department of Chemistry, Clemson University, Clemson, SC 29634, USA
}

Received December 4, 2006

\begin{abstract}
Using Raman scattering and optical birefringence we have investigated a low-temperature phase transition in single crystal of the two-dimensional $\mathrm{Na}_{5} \mathrm{RbCu}_{4}\left(\mathrm{AsO}_{4}\right)_{4} \mathrm{Cl}_{2}$. Phonon anomalies point to a first order nature of the transition. The observed transition is most probably related to a order-disorder transition of the $\mathrm{Rb}$ ion positions along the $z$ axis within the ionic framework of mixed alkali metal chloride lattices.
\end{abstract}

PACS: 64.60.-i General studies of phase transitions;

63.20.-e Phonons in crystal lattices;

78.20.Fm Birefringence;

78.30.-j Infrared and Raman spectra (for vibrational states in crystals and disordered systems).

Keywords: structural phase transition, optical birefringence, Raman scattering.

A large part of the motivation to search and study new families of materials with small spin and low dimensionality is based on the discovery of high-temperature superconductivity in the cuprates. Following this line two-dimensional systems with pronounced magnetic fluctuations, competing interactions and structural instabilities have been discovered and investigated $[1,2]$. Recently a new salt-inclusion copper arsenate $\mathrm{Na}_{5} \mathrm{RbCu}_{4}\left(\mathrm{AsO}_{4}\right)_{4} \mathrm{Cl}_{2}$ with a remarkable crystal structure was synthesized using a conventional solid-state reaction [3]. This compound exhibits a composite structure of alternating magnetic and insulating layers. The magnetic layer contains $\left[\mathrm{Cu}_{4} \mathrm{O}_{12}\right]^{16-}$ tetrameric units with $\mathrm{Cu}_{4} \mathrm{O}_{4}$ magnetic cores, which are connected by $\mathrm{AsO}_{4}$ bridging units. The copper valence state is $2+$, so the $\mathrm{Cu}$ ions are magnetic with spin $1 / 2$. The insulating layer consists of an ionic framework of mixed alkali metal chloride lattices and rarely seen $\mathrm{Na}_{6} \mathrm{O}_{8}$ clusters. Also the room tempera- ture crystal structure of $\mathrm{Na}_{5} \mathrm{RbCu}_{4}\left(\mathrm{AsO}_{4}\right)_{4} \mathrm{Cl}_{2}$ was reported as orthorhombic (space group $F m m m, Z=2$ ) [4]. The low-temperature crystal structure, which may have crucial implications for the distinct magnetic order, has not been determined until now. In the Ref. 5 two structural phase transitions around 74 and $110 \mathrm{~K}$ seen by ${ }^{87} \mathrm{Rb}$ nuclear magnetic resonance were reported but their nature was not clarified.

To get more insight into the structural phase transitions in $\mathrm{Na}_{5} \mathrm{RbCu}_{4}\left(\mathrm{AsO}_{4}\right)_{4} \mathrm{Cl}_{2}$ we performed Raman scattering (RS) and birefringence experiments. Experiments on the visual observations of the domain structure formed in the vicinity of the structural phase transition were carried in parallel.

RS measurements were performed in quasi-backscattering geometry with the excitation line $\lambda=514.5 \mathrm{~nm}$ of an $\mathrm{Ar}^{+}$laser. The laser power of $5 \mathrm{~mW}$ was focused to a $0.1 \mathrm{~mm}$ diameter spot on the sample surface. Raman spec- 
tra measured with different laser powers showed that the chosen power is sufficiently small to ensure a negligible heating of the sample. Spectra of the scattered radiation were collected by a DILOR-XY triple spectrometer and recorded by a nitrogen cooled charge-coupled device detector with a spectral resolution of $\sim 1 \mathrm{~cm}^{-1}$. Single crystals are transparent, blue, and plate like with plate surface perpendicular to $b$ axis. Typical crystal dimensions are $3 \mathrm{~mm} \times 1 \mathrm{~mm} \times 200 \mu \mathrm{m}$.

The linear optical birefringence

$$
\Delta n=\delta \lambda / 2 \pi t,
$$

where $\delta$ is the phase shift between the linearly polarized optical eigenmodes of the crystal, and $t$ is the thickness of the crystal, was measured in an optical helium cryostat. In the experiments a quarterwave plate was used as the compensator to measure the birefringence. In this case, $\delta$ was defined accurate to $2 \pi k$ where $k=0,1,2, \ldots$ A filament lamp and an interference filter with a pass band of $11 \mathrm{~nm}$ width were used as a light source. Measurements were carried out with the wavelength of $\lambda=515 \mathrm{~nm}$.

For the visual observations of the domain structure formed in the vicinity of the structural phase transition a polarizing optical microscope was used.

The group-theoretical analysis of the $\Gamma$-point Raman-active phonon modes for the orthorhombic $\mathrm{Na}_{5} \mathrm{RbCu}_{4}\left(\mathrm{AsO}_{4}\right)_{4} \mathrm{Cl}_{2}$ is presented in Table 1. Of the total $186 \Gamma$-point phonon modes, 99 modes $\left(29 A_{g}+25 B_{1 g}+\right.$ $+21 B_{2 g}+24 B_{3 g}$ ) are Raman-active. In Fig. 1, we show the polarized Raman spectra of $\mathrm{Na}_{5} \mathrm{RbCu}_{4}\left(\mathrm{AsO}_{4}\right)_{4} \mathrm{Cl}_{2}$ at room temperature. The sharpness of observed lines is evidence for the high quality of the studied samples. Experimental values of the Raman frequencies are summarized in Table 2. The number of Raman-allowed modes (99) is

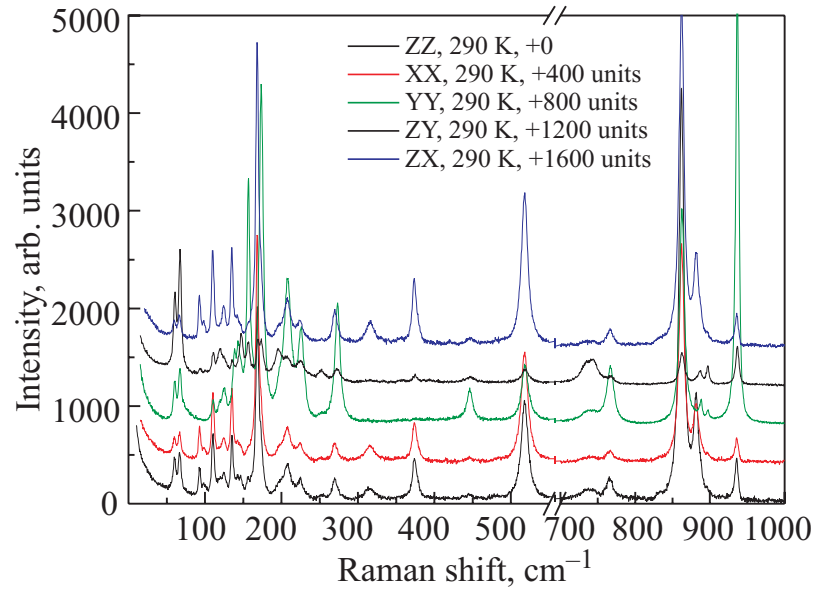

Fig. 1. Polarized room temperature Raman spectra of a $\mathrm{Na}_{5} \mathrm{RbCu}_{4}\left(\mathrm{AsO}_{4}\right)_{4} \mathrm{Cl}_{2}$ single crystal.

three times larger than the number of experimentally observed lines (33) in the frequency region of $0-1000 \mathrm{~cm}^{-1}$. It is practically impossible to further analyze the vibrations in such a low-symmetry structure with a large number of partially overlapping Raman-active modes at room temperature.

The most characteristic Raman features related to the structural phase transition with decreasing temperature are the sudden splitting of some phonon modes and the appearance of new modes. In Fig. 2 an examples of observed changes in the Raman spectra with temperature are shown. Intuitively the character of changes allows us to suggest a first order nature of the phase transition. Earlier the structural phase transition at $T=74 \mathrm{~K}$ in $\mathrm{Na}_{5} \mathrm{RbCu}_{4}\left(\mathrm{AsO}_{4}\right)_{4} \mathrm{Cl}_{2}$ was related to a change of the local symmetry at the $\mathrm{Rb}$ sites [5].

Table 1. Wyckoff notations and Raman irreducible representations for the atoms in orthorhombic $\mathrm{Na}_{5} \mathrm{RbCu}_{4}\left(\mathrm{AsO}_{4}\right)_{4} \mathrm{Cl}_{2}\left(\mathrm{space}_{\mathrm{group}}\right.$ at room temperature is Fmmm N69 [3])

\begin{tabular}{c|c|c}
\hline \hline Atom & Wyckoff \\
notation & $\Gamma_{\text {Raman }}$ \\
\hline \hline $\mathrm{Rb}(1)^{*}$ & $8 g$ & $1 A_{g}(x x, y y, z z)+1 B_{1 g}(x y)+1 \mathrm{~B}_{2 g}(x z)+0 B_{3 g}(y z)$ \\
$\mathrm{Rb}(2)^{*}$ & $16 n$ & $2 A_{g}(x x, y y, z z)+1 B_{1 g}(x y)+2 B_{2 g}(x z)+1 B_{3 g}(y z)$ \\
$\mathrm{As}(1), \mathrm{O}(3), \mathrm{O}(4)$ & $16 o$ & $2 A_{g}(x x, y y, z z)+2 B_{1 g}(x y)+1 B_{2 g}(x z)+1 B_{3 g}(y z)$ \\
$\mathrm{As}(2), \mathrm{O}(1), \mathrm{O}(2)$ & $16 m$ & $2 A_{g}(x x, y y, z z)+1 B_{1 g}(x y)+1 B_{2 g}(x z)+2 B_{3 g}(y z)$ \\
$\mathrm{Cu}, \mathrm{O}(5), \mathrm{O}(6)$ & $32 p$ & $3 A_{g}(x x, y y, z z)+3 B_{1 g}(x y)+3 B_{2 g}(x z)+3 B_{3 g}(y z)$ \\
$\mathrm{Na}(1), \mathrm{Cl}(1)$ & $8 h$ & $1 A_{g}(x x, y y, z z)+1 B_{1 g}(x y)+0 B_{2 g}(x z)+1 B_{3 g}(y z)$ \\
$\mathrm{Na}(2)$ & $16 n$ & $2 A_{g}(x x, y y, z z)+1 B_{1 g}(x y)+2 B_{2 g}(x z)+1 B_{3 g}(y z)$ \\
$\mathrm{Na}(3)$ & $16 k$ & $1 A_{g}(x x, y y, z z)+2 B_{1 g}(x y)+1 B_{2 g}(x z)+2 B_{3 g}(y z)$ \\
$\mathrm{Cl}(2)$ & $8 d$ & $0 A_{g}(x x, y y, z z)+0 B_{1 g}(x y)+0 B_{2 g}(x z)+0 B_{3 g}(y z)$ \\
\hline \hline
\end{tabular}

* According to the data [1] the refined occupancies for $\mathrm{Rb}(1)$ and $\mathrm{Rb}(2)$ are 45 and $27.5 \%$, respectively, to have a total of $100 \%$ occupancy with two $\mathrm{Rb}$ ions per primitive cell. Total: $\Gamma_{\text {Raman }}=29 A_{g}(x x, y y, z z)+25 B_{1 g}(x y)+21 B_{2 g}(x z)+24 B_{3 g}(y z)$. 
Table 2. Energy of the measured Raman modes in $\mathrm{Na}_{5} \mathrm{RbCu}_{4}\left(\mathrm{AsO}_{4}\right)_{4} \mathrm{Cl}_{2}$ at room temperature in wave numbers $\left(\mathrm{cm}^{-1}\right)$

\begin{tabular}{|c|c|c|c|c|}
\hline$a a$ & $c c$ & $c a$ & $b b$ & $c b$ \\
\hline 60.1 & 60.1 & 60.1 & 60.1 & $60.1^{*}$ \\
\hline 67.0 & 67.0 & 67.0 & 67.0 & 67.0 \\
\hline 92.9 & 92.9 & 92.9 & & 92.9 \\
\hline 98.6 & 98.6 & $98.6^{*}$ & & 98.6 \\
\hline \multirow[t]{2}{*}{110.1} & 110.1 & 110.1 & 110.1 & 110.1 \\
\hline & & & 119.2 & $119.2^{*}$ \\
\hline 124.5 & 124.5 & $124.5 *$ & 124.5 & 124.5 \\
\hline \multirow[t]{2}{*}{135.4} & 135.4 & 135.4 & & 135.4 \\
\hline & & & 138.3 & \\
\hline 142.9 & 142.9 & 142.9 & $142.9^{*}$ & 142.9 \\
\hline 147.3 & 147.3 & 147.3 & 147.3 & $147.3^{*}$ \\
\hline 156.8 & 156.8 & 156.8 & $156.8^{*}$ & 156.8 \\
\hline 168.5 & 168.5 & $168.5^{*}$ & 168.9 & 168.5 \\
\hline 173.5 & 173.5 & 173.5 & $173.5^{*}$ & 173.5 \\
\hline 196.8 & 196.8 & 196.8 & 196.8 & 196.8 \\
\hline 207.7 & 207.7 & 207.7 & $207.7^{*}$ & 207.7 \\
\hline 224.7 & 224.7 & 224.7 & $224.7 *$ & 224.7 \\
\hline 252.3 & 252.3 & 252.3 & 252.3 & 252.3 \\
\hline \multirow[t]{2}{*}{269.9} & 269.9 & 269.9 & & \\
\hline & & & $273.3^{*}$ & 273.3 \\
\hline 315.9 & 315.9 & 315.9 & & \\
\hline \multirow[t]{2}{*}{374.3} & 374.3 & 374.3 & & \\
\hline & & & $445.7^{*}$ & \\
\hline 518.2 & 518.2 & 518.2 & 518.2 & $518.2^{*}$ \\
\hline 734.5 & 734.5 & 734.5 & 734.5 & $734.5^{*}$ \\
\hline 744.8 & 744.8 & 744.8 & 744.8 & $744.8^{*}$ \\
\hline 766.6 & 766.6 & 766.6 & $766.6^{*}$ & 766.6 \\
\hline 832.6 & 832.6 & 832.6 & & \\
\hline 862.3 & 862.3 & 862.3 & 862.3 & 862.3 \\
\hline \multirow[t]{3}{*}{881.9} & 881.9 & 881.9 & & \\
\hline & & & 887.1 & 887.1 \\
\hline & & 897.3 & 897.3 & 897.3 \\
\hline \multirow[t]{2}{*}{935.5} & 935.5 & 935.5 & & \\
\hline & & & 936.6 & 936.6 \\
\hline
\end{tabular}

* most intensive lines

Visual studies and investigation of temperature dependence of birefringence allows us to define more precisely the order of the structural phase transition in $\mathrm{Na}_{5} \mathrm{RbCu}_{4}\left(\mathrm{AsO}_{4}\right)_{4} \mathrm{Cl}_{2}$. In Fig. 3 the temperature de-

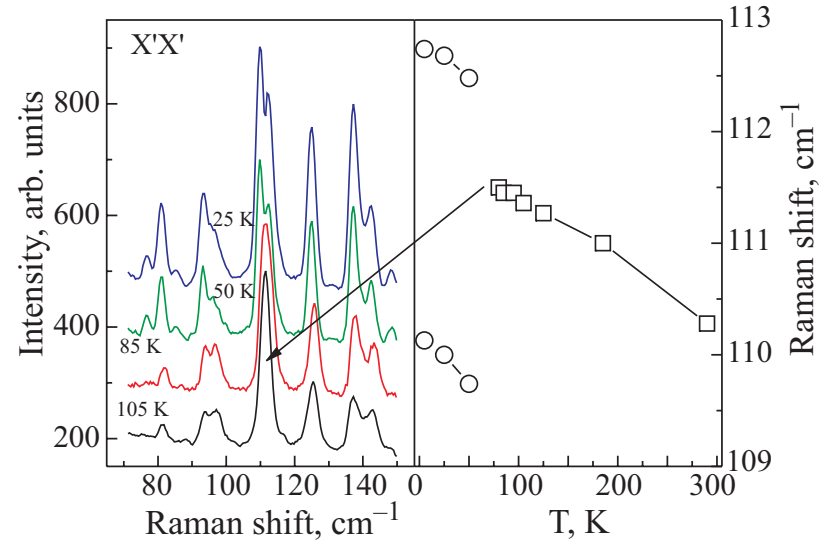

Fig. 2. Temperature dependence of Raman spectra of $\mathrm{Na}_{5} \mathrm{RbCu}_{4}\left(\mathrm{AsO}_{4}\right)_{4} \mathrm{Cl}_{2}$ and temperature dependence of phonon energies.

pendence of birefringence is presented. Our experiments point to a small increase of $\delta$ with decreasing temperature up to $174 \mathrm{~K}$. A further decreasing of temperature lead to $\delta$ decreasing and at $T=73 \mathrm{~K}$ there is a sharp increase of $\delta$ which corresponds to a first order structural phase transition. In the low-temperature phase the axes of optical indicatrix of the crystal (inset $b$ in Fig. 3) are turned by an angle of $45^{\circ}$ relative to axes of optical indicatrix before the phase transition (inset $a$ in Fig. 3). At temperatures below the phase transition the value of $\delta$ changes weakly. By substituting $t=200 \mu \mathrm{m}, \delta=70.4^{\circ}, \lambda=515 \mathrm{~nm}$ into Eq. (1), we obtain $\Delta n \approx 10^{-3}$ at $T=32 \mathrm{~K}$. On heating the phase transition occurs at $T=83 \mathrm{~K}$. Thus, the temperature hysteresis of birefringence by value of about $10 \mathrm{~K}$ is observed at the first order phase transition in the crystal of $\mathrm{Na}_{5} \mathrm{RbCu}_{4}\left(\mathrm{AsO}_{4}\right)_{4} \mathrm{Cl}_{2}$.

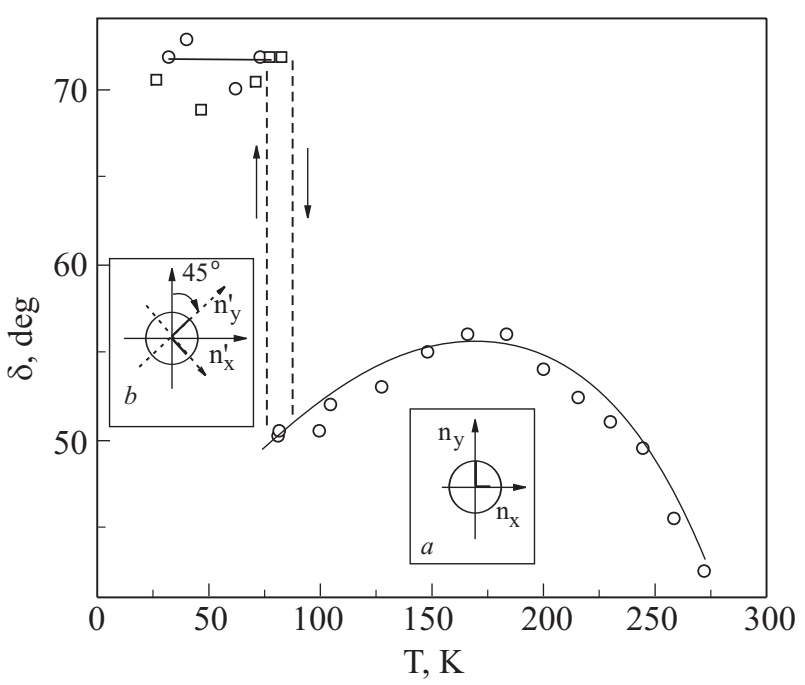

Fig. 3. Temperature dependence of $\delta$ in the $\mathrm{Na}_{5} \mathrm{RbCu}_{4}\left(\mathrm{AsO}_{4}\right)_{4} \mathrm{Cl}_{2}$ single crystal. Insets show sections of the optical indicatrix of the crystal before $(a)$ and after $(b)$ structural phase transition. 


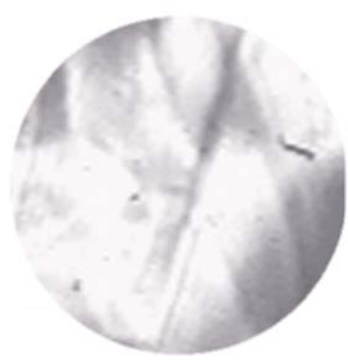

$a$

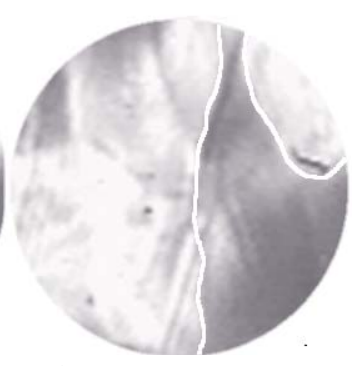

$b$

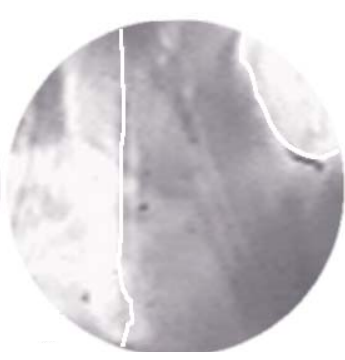

$c$

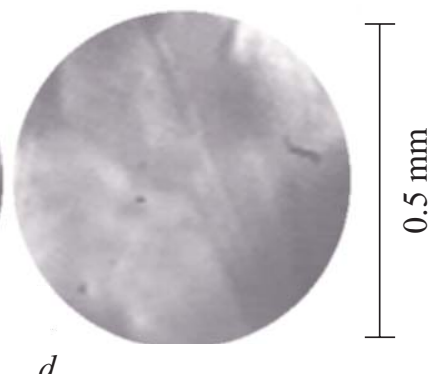

d

Fig. 4. The two-phase domain structure formed at structural phase transition in the crystal of $\mathrm{Na}_{5} \mathrm{RbCu}_{4}\left(\mathrm{AsO}_{4}\right)_{4} \mathrm{Cl}_{2}$. The temperature varies from $82.5 \mathrm{~K}(a)$ up to $T=72.5 \mathrm{~K}(d)$. Light line shows the border between crystal phases $(b, c)$.

As it is mentioned above the first order phase transition in $\mathrm{Na}_{5} \mathrm{RbCu}_{4}\left(\mathrm{AsO}_{4}\right)_{4} \mathrm{Cl}_{2}$ is related to a sharp increase of birefringence and to a rotation of the optical indicatrix axes by $45^{\circ}$. Due to this it is possible to investigate the two-phase crystal domain structure formed at the phase transition visually in polarized light. In Fig. $4, a$ the image of the investigated sample before phase transition $(T=82.5 \mathrm{~K})$ is presented. In this photo dark areas are due to defects of the sample. With decreasing temperatures the new crystal phase forms in the crystal (Fig. 4,b). In this photo the boundary between crystal phases is shown by a light line. With further decreasing of temperature the area of a new phase increases (Fig. 4,c). And at last, at $T=72.5 \mathrm{~K}$, the phase transition is completed (Fig. 4, $d$ ).

In order to discuss the possible symmetry of the new phase one should mentioned that due to the large size of the primitive cell with a large number of ions we can not expect a drastic change in the primitive cell volume under structural phase transition. The minor change of Raman spectra evidences also that not so much lattice degrees of freedom are affected by structural phase transition. Moreover, due to this reason the space group of low-temperature phase should demonstrate a subgroup connection with Fmmm space group of the high-temperature phase. The $45^{\circ}$ rotation of the optic indicatrix and the absence of optical activity in low-temperature phase give some hints about this connection.

Some candidate for the driving force of the structural phase transition can be an uncertainty in the Rb ions positions which is defined at room temperature [3]. Interestingly, that high symmetry $8 g \mathrm{Rb}(1)$ position with occupancy $45 \%$ and main representative coordinates $(0.1521(5), 0,0)$ is refined isotropically, while the low symmetry $16 n \mathrm{Rb}(2)$ position with occupancy $27.5 \%$ and main representative coordinates $(0.1492(3), 0,0.0443(4))$ is refined anisotropically [3]. The last one demonstrates the tendency for $\mathrm{Rb}$ ions to be shifted along $z$ axis. However, the complete occupancy of the $16 n$ position is impossible because it violates the allowed number of $\mathrm{Rb}$ ions per primitive cell. While the shift of $\mathrm{Rb}$ ions in the fully occupied $8 g$ position along $z$ or $y$ directions leads to the lowering of symmetry from orthorhombic to monoclinic. Supposing a first order structural phase transition from low-temperature to high-temperatures as a transition from order to disorder at the $\mathrm{Rb}$ sites one can chose the highest subgroup of the space group Fmmm that conserves inversion center, number of structural units per cell, and includes the shift of $\mathrm{Rb}$ ions along $z$ axis. This subgroup is $C 2 / m$ space group with unique axis along $b$ direction of the high-temperature phase space group Fmmm [6]. One of the in-plane $C 2 / m$ axes coincides with $x$ axis of the Fmmm group, while another one should be directed along $(-1,0,1)$. In the absence of monoclinic distortions, due to small rhombicity, it rotates on $45.36^{\circ}$ from $z$ axis of the high-temperature phase. This angle slightly deviates from $45.36^{\circ}$ in the presence of monoclinic distortions.

Raman data also support the proposed model. The apparent changes of spectra occur in the low-frequency region, to which the heaviest ions ( $\mathrm{Rb}$ and $\mathrm{As}$ ) mainly contribution. Whereas, the high-frequency region which is formed by the vibrations of light complexes like $\mathrm{Na}_{6} \mathrm{O}_{8}$ and others does not demonstrate anomalies.

In conclusion, using Raman scattering and optical birefringence we have shown that first-order phase transition in $\mathrm{Na}_{5} \mathrm{RbCu}_{4}\left(\mathrm{AsO}_{4}\right)_{4} \mathrm{Cl}_{2}$ can be related to a orderdisorder transition of the $\mathrm{Rb}$ ion positions along the $z$ axis.

This work was partially supported by the ESF (ESF-HFM) and the DFG (Priority program «Molecular Magnetism»).

1. H. Kageyama, J. Phys. Soc. Jpn. 69, 65 (2000).

2. P. Lemmens, G. Güntherodt, and C. Gross, Phys. Rep. 375, 1 (2003); P. Lemmens and P. Millet, in «Quantum Magnetism», U. Schollwцck, J. Richter, B.J.J. Farell, and R.F. Bishop (eds.), Springer, Heidelberg, «Lecture Notes in Physics» (2004).

3. Shiou-Jyh Hwu, Mutlu Ulutagay-Kartin, Jeffrey A. Clayhold, Richard Mackay, Tina A. Wardojo, Charles J. O'Con- 
nor, and Mariusz Krawiec, J. Am. Chem. Soc. 124, 12404 (2002).

4. J.A. Clayhold, M. Ulutagay-Kartin, S.-J. Hwu, H.-J. Koo, M.-H. Whangbo, A. Voigt, and K. Eaiprasertsak, Phys. Rev. B66, 052403 (2002).
5. R. Stern, I. Heinmaa, A. Kriisa, E. Joon, S. Vija, J. Clayhold, M. Kartin-Ulutagay, X. Mo, W. Queen, and S.-J. Hwu, arXiv:cond-mat/051272.

6. V.E. Naish and V.N. Syromyatnikov, Subgroups of the Space Groups-I. Subgroups with Preservation Unit Cell, preprint No 2371-76, Dep. (Institute of Metal Physics, RAS, 1976) (in Russian). 